\title{
Pédagogie universitaire et didactique des mathématiques
}

Colette Picard

\section{(2) OpenEdition \\ 12 Journals}

Édition électronique

URL : https://journals.openedition.org/ripes/243

DOI : 10.4000/ripes.243

ISSN : 2076-8427

Éditeur

Association internationale de pédagogie universitaire

Référence électronique

Colette Picard, "Pédagogie universitaire et didactique des mathématiques », Revue internationale de pédagogie de l'enseignement supérieur [En ligne], 25(2) | 2009, mis en ligne le 14 septembre 2009, consulté le 21 septembre 2021. URL : http://journals.openedition.org/ripes/243 ; DOI : https://doi.org/ $10.4000 /$ ripes. 243

Ce document a été généré automatiquement le 21 septembre 2021.

Article L.111-1 du Code de la propriété intellectuelle. 


\title{
Pédagogie universitaire et didactique des mathématiques
}

\author{
Colette Picard
}

1 L'enseignement dans un contexte universitaire nous confronte à différents défis. Ainsi doit-on aider les étudiants à acquérir des compétences sans pouvoir, pour autant, leur offrir un contexte d'enseignement où celles-ci peuvent se développer de manière authentique. C'est le cas dans le cours Difficultés d'apprentissage en mathématiques,quifait partie du cursus du baccalauréat en éducation préscolaire et en enseignement primaire de l'Université du Québec en Abitibi-Témiscamingue.

2 Comme responsable de ce cours, nous étions soucieuse d'offrir à nos étudiants, futurs enseignants au préscolaire et au primaire, un environnement pédagogique favorable à l'émergence des compétences professionnelles requises. Nous avons donc choisi d'orienter notre approche pédagogique vers l'apprentissage par problèmes. Notre intérêt pour cette approche réside dans le fait que, à de nombreuses reprises, nous avons observé que les étudiants inscrits à notre cours Difficultés d'apprentissage en mathématiques éprouvaient des difficultés à enseigner selon une perspective socioconstructiviste, même s'ils avaient d'abord reçu les connaissances théoriques qui permettent a priori de le faire. En effet, dans leur enseignement, au lieu d'exploiter des stratégies favorisant la construction de concepts par les élèves eux-mêmes, ils utilisaient principalement des explications et des démonstrations. Ainsi, ils éprouvaient des difficultés à planifier des activités qui placent l'élève dans un contexte l'obligeant à réorganiser ses connaissances pour résoudre un problème. Leurs activités pédagogiques étaient essentiellement centrées sur leur enseignement au détriment de l'implication des élèves dans leur propre apprentissage. Il devenait donc impératif de trouver un moyen pour amener nos étudiants à mieux appréhender et maitriser l'impact de l'apprentissage par problèmes sur la réussite des élèves du primaire.

3 Nous émettons l'hypothèse que les étudiants ne peuvent pas intégrer et ensuite transposer une conception constructiviste de l'apprentissage puisqu'ils ne l'ont pas vécue eux-mêmes dans leur parcours scolaire. De plus, l'enseignement universitaire traditionnel, qui privilégie l'enseignement frontal et la transmission de contenus, ne 
leur permet pas non plus d'expérimenter ni d'observer d'autres approches pédagogiques. Ils sont en quelque sorte enfermés dans un cadre transmissif acquis par modelage où l'on reproduit ce qu'on a vécu soi-même comme apprenant.

4 Cet article fait état d'un changement de pratique dans la manière de former les étudiants et de les préparer à adopter différentes approches pédagogiques. Nous commencerons par exposer plus en détail l'élément déclencheur et le contexte qui a présidé à notre décision de renouveler la formation offerte aux étudiants et notre propre approche pédagogique. Nous mettrons en évidence comment l'apprentissage par problèmes nous a présenté différentes avenues pour favoriser le développement des compétences requises chez nos étudiants. Nous exposerons ensuite notre démarche d'implantation avec une analyse des conditions qui favorisent ce type de pédagogie, des difficultés rencontrées lors de ce changement de pratique ainsi que des retombées pour les étudiants et la professeure en charge de l'enseignement.

\section{L'élément déclencheur}

5 Nos étudiants sont inscrits au baccalauréat en éducation au préscolaire et en enseignement primaire et en sont à leur troisième année de formation. Ils se présentent au cours Difficultés d'apprentissage en mathématiques après avoir réussi trois cours préalables: 1) Les mathématiques comme objet d'apprentissage; 2) Didactique des mathématiques I; et 3) Didactique des mathématiques II. Lors de tous ces cours, ils sont constamment sensibilisés à l'importance du socioconstructiviste dans la construction des séquences didactiques. Malgré cela, plusieurs étudiants manifestent de la difficulté à tenir compte de ces éléments dans l'élaboration de leurs situations d'enseignementapprentissage. Lorsqu'ils donnent une leçon, par exemple, ils se rapportent souvent à la façon dont eux-mêmes ont appris le concept lorsqu'ils étaient au primaire, il y a une dizaine d'années, plutôt que d'en favoriser la construction par l'élève lui-même, comme le suggère le programme de formation de l'école québécoise.

6 Le socioconstructivisme occupe une place importante en didactique des mathématiques dans la formation des maîtres. Or, enseigner selon une telle perspective implique que l'enseignant en devenir soit au fait des processus d'apprentissage des élèves. Les travaux de Piaget, entre autres, sont d'un grand apport pour aborder ces questions, en particulier lorsqu'il argumente que l'enfant apprend face à un déséquilibre où ses schèmes antérieurs ne sont plus suffisants pour résoudre le problème auquel il est confronté (Jonnaert \& Vander Borght, 1999 ; Piaget, 1975). Cette observation implique qu'il faut renoncer à transmettre des connaissances sur les concepts mais plutôt créer des situations qui permettent à l'enfant de prendre conscience de ses limites et qui l'amènent à développer d'autres connaissances en respectant une certaine progression dans le niveau d'abstraction du concept présenté. Or, cette approche de l'apprentissage est considérablement différente de ce qu'ont vécu nos étudiants lors de leur scolarité. Nous observons que leur propre expérience de l'enseignement des mathématiques l'emporte sur les connaissances pédagogiques qu'ils acquièrent à propos de la didactique des mathématiques. Cette observation n'est pas spécifique à notre situation et d'autres l'ont aussi relevée (Roy, 2007). Le défi pour nos étudiants, futurs enseignants, consiste à se détacher de leur vécu d'apprenant pour intégrer et surtout mettre en œuvre de nouvelles pratiques qui se distancient de celles qu'ils ont connues. 
7 Nous souscrivons à la définition de Langevin \& Bruneau (2000) qui conçoivent l'apprentissage comme une construction personnelle prenant appui sur les connaissances antérieures de l'étudiant. Le principe de la cohérence pédagogique veut, dès lors, que nous proposions à nos étudiants des situations où ils doivent intervenir selon une perspective socioconstructiviste pour ensuite utiliser ce vécu comme point d'ancrage. Cette option nous apparaît intéressante, d'autant plus que Langevin \& Bruneau nous rappellent que le professeur doit mettre à jour les connaissances et les expériences antérieures des étudiants, leur permettant ainsi d'accéder au nouveau savoir et de le raccrocher à l'ancien. Toutefois, comment proposer des expériences nouvelles aux étudiants pour faciliter le développement de compétences liées à l'enseignement des mathématiques dans une perspective socioconstructiviste et, de surcroît, comment innover dans un contexte universitaire traditionnel?

\section{L'intérêt pour l'apprentissage par problème (APP)}

8 Ces questionnements nous ont conduite à revoir notre type de pédagogie et nos réflexions pédagogiques ont évolué autour de la question suivante: l'apprentissage par problèmes pourra-t-il nous permettre d'atteindre les intentions pédagogiques de notre cours ? Guilbert \& Ouellet (1997) décrivent l'apprentissage par problèmes comme « un processus de résolution de problèmes complexe où les participants, regroupés par équipes, travaillent ensemble à chercher des informations et à résoudre un problème réel ou réaliste proposé de façon à développer des compétences de résolution de problèmes et à faire en même temps des apprentissages de contenu (p67)». Selon cette approche, l'accent est davantage mis sur le processus d'apprentissage que sur le processus d'enseignement où enseigner revient pour l'enseignant à transmettre, à communiquer tandis qu'apprendre signifie construire pour l'apprenant. Or, comme le rappellent si bien Langevin \& Bruneau (2000), "Ce n'est pas parce que l'enseignant expose ce qu'il sait que l'étudiant devient automatiquement quelqu'un (...) qui apprend (p12)».

9 L'efficacité pédagogique de l'apprentissage par problèmes a fait l'objet de nombreuses études. Delorme, Jean \& Des Marchais (1987) reconnaissent la validité de ce type de pédagogie en ces termes :

«(...) il a été démontré que c'est une méthode pédagogique valide. En effet, elle semble répondre à certaines déficiences de l'enseignement traditionnel. Des études contrôlées tendent à démontrer qu'elle est plus efficace que l'enseignement habituel basé sur la leçon magistrale. De plus, elle possède une validité de construit. En effet, des recherches récentes sur l'encodage de l'information dans la mémoire à long terme montrent que plus la situation d'apprentissage d'une connaissance se rapproche de la situation où cette connaissance sera utilisée, plus on a de chance que cette connaissance soit non seulement retenue mais aussi disponible et utilisée à bon escient par la suite (p281). »

Compte tenu de notre préoccupation et de notre intention d'améliorer les dispositifs de formation de nos étudiants, tout porte à croire que l'apprentissage par problèmes offre des options intéressantes pour assurer un apprentissage en profondeur (Larue \& Cossette, 2006). 


\section{Méthode de travail et dispositif de formation}

11 Précisons d'abord que notre examen de la littérature sur l'apprentissage par problèmes fait appel à l'usage de différents types de problèmes pour planifier une séquence didactique ou un dispositif de formation : les problèmes réels, les problèmes fictifs et les problèmes réalistes. Or, parmi les recherches qui ont été portées à notre attention, aucune ne fait état d'interventions directes, conduites durant la période même du cours, et ce sur des sujets éprouvant effectivement des difficultés d'apprentissage concernant les savoirs essentiels en mathématiques. Cependant, même les problèmes les plus réels ne permettent pas de développer les mêmes compétences que celles que nous mobilisons lorsque nous travaillons directement avec un enfant. Dans la situation présente, l'étudiant s'engage dans un plan d'intervention qui va s'échelonner sur six semaines avec le même enfant. L'implication de la professeure, tout au long des interventions, représente un élément particulier de ce projet. Elle anime les discussions des équipes sur les différents problèmes rencontrés, établit les liens avec la théorie, valide la planification de chaque participant et supervise les interventions directes avec les enfants. Elle assure aussi le suivi avec les enseignants des différentes écoles primaires concernées par le projet.

Ainsi, sur les 45 heures que totalise le cours, six seront réalisées directement à l'école primaire. Ces rencontres débutent au quatrième cours, donc après 12 heures d'enseignement. Durant cette période, l'étudiant valide sa maitrise des concepts de base en mathématiques, mobilise ses connaissances sur la didactique, se familiarise avec le plan d'intervention et s'initie au pilotage d'une activité d'enseignement auprès d'un élève en difficulté.

$\mathrm{Au}$ quatrième cours, la professeure accueille ses étudiants directement à l'école primaire pour superviser les activités qu'ils réaliseront avec les enfants. Par la suite, les étudiants réintègrent la salle de cours dans les locaux de l'université. Le cours se poursuit en tenant compte de ce que nous venons de vivre tant en ce qui se rapporte aux contenus liés à l'intervention qu'à ceux concernant la théorie. Les six cours qui suivent se déroulent ainsi. Les derniers cours de la session sont réservés à la mise en commun des différentes problématiques et à la synthèse des apprentissages en vue de l'examen final auquel les étudiants sont soumis dans le cadre de l'évaluation des apprentissages.

14 Il faut souligner que cette situation présente plusieurs contraintes, dont la plus importante est l'incompatibilité des horaires entre les deux ordres d'enseignement. En effet, les établissements scolaires de niveau primaire fonctionnent sur des horaires de neuf jours, ce qui signifie que le lundi peut être un jour 1 , un jour 4 , etc. Chaque jour respecte un horaire spécifique différent de celui de chaque autre jour. Cette façon d'aménager le temps était nécessaire pour répartir équitablement le temps réservé à l'enseignement de l'anglais et de l'éducation physique, qui ne sont pas sous la responsabilité du titulaire de la classe. Cela implique donc des modifications à l'horaire des classes du primaire concernées puisque notre cours doit toujours avoir lieu au même moment, à chaque semaine (par exemple, le lundi à 13 heures). Malgré tout, le projet suscite suffisamment d'intérêt pour que les deux écoles contactées acceptent de collaborer à sa réalisation.

15 Les élèves sont sélectionnés par les enseignantes dans les groupes de $3^{\mathrm{e}}$ cycle, soit des enfants ayant 11 ou 12 ans. Ces élèves sont identifiés parce qu'ils éprouvent de réelles 
difficultés en mathématiques, notamment en ce qui concerne les opérations sur les nombres naturels, les fractions et les décimaux. Nous avons choisi ces notions, d'une part, parce qu'elles sont reconnues pour le degré de difficulté qu'elles représentent tant pour l'enseignant que pour les élèves et, d'autre part, parce qu'elles constituent aussi un réel défi pour nos étudiants.

Dans un premier temps, la présentation du projet aux étudiants génère de l'enthousiasme, mais elle provoque aussi beaucoup d'anxiété. D'entrée de jeu, ils ne se sentent pas en mesure d'intervenir efficacement auprès d'élèves présentant de réelles difficultés. Cependant, rapidement, ils prennent conscience des exigences de la tâche et développent différentes compétences au fur et à mesure que se déroule le cours et que la pratique donne sens à la théorie. Notons que la taille des groupes d'étudiants varie significativement d'une session à l'autre. Ainsi, nous avons proposé notre projet à des étudiants universitaires qui faisaient partie d'un groupe de 12 ; nous l'avons aussi présenté à un groupe de 40 étudiants. Néanmoins, d'une façon générale, nos groupes sont composés d'environ 30 participants.

\section{Notre analyse du projet}

17 Il ne faut pas perdre de vue que même si le concept d'apprentissage par problèmes a été introduit au Canada en 1965, c'est seulement en 1980 que la Faculté de médecine de l'Université de McMaster l'a incorporé dans ses plans d'études (Larue \& Cossette, 2006). Lorsque nous avons commencé à modifier notre propre pratique enseignante en 1996, la thématique n'était pas aussi documentée qu'aujourd'hui, et nous disposions de peu d'informations pour implanter notre projet. Cependant, nous pouvons maintenant examiner notre démarche à partir d'une littérature florissante et alimenter, à notre tour, la réflexion d'autres pédagogues grâce à notre expérience. L'analyse qui suit porte sur le type de problèmes choisis, les conditions favorables, le pilotage du changement, les difficultés rencontrées, les retombées ainsi que les limites du projet.

\subsection{Type de problèmes}

18 Comme nous l'avons vu, plus la situation se rapprochera de la situation réelle, plus les apprentissages seront durables et plus ils seront transposés à bon escient (Delorme, Jean \& Des Marchais, 1987). C'est ce que nous voulons offrir à nos étudiants. Nous leur proposons donc d'intervenir directement auprès d'un élève en difficulté d'apprentissage en mathématiques. Chaque étudiant est jumelé à un seul élève. Il reçoit une fiche lui donnant le nom de l'enfant ainsi qu'une analyse sommaire de ses difficultés concernant les savoirs essentiels en mathématiques. Les étudiants doivent, lors de la première rencontre, valider les difficultés, établir la liste des préalables et proposer un plan d'intervention qui doit tenir compte des connaissances antérieures. Par la suite, ils doivent présenter les concepts dans une perspective constructiviste, évaluer la progression de l'élève tout en respectant ses intérêts et en créant une relation propice à l'apprentissage.

Précisons que le fait de travailler directement avec des enfants en difficulté d'apprentissage dans les écoles nous place dans une situation délicate. En effet, même si nos étudiants sont en formation, nous ne pouvons ignorer que leurs compétences et la 
qualité des interventions influencent la réussite scolaire des élèves. C'est ce que nos partenaires du milieu scolaire nous ont rapporté au cours des dix dernières années.

\subsection{Conditions favorables}

20 point de vue du dispositif de formation, nos étudiants en sont à leur troisième année de scolarité universitaire et possèdent une expertise concernant l'enseignement des mathématiques: d'une part, ils ont eu la possibilité de développer des habiletés liées à la planification et à l'évaluation et, d'autre part, ils ont aussi développé des habiletés pour établir une relation pédagogique avec un élève. Le projet respecte les intentions pédagogiques du cours, c'est-à-dire permettre aux étudiants d'élaborer et de piloter des situations d'enseignement-apprentissage auprès d'élèves en difficulté d'apprentissage.

Ensuite, dans un contexte plus large, deux écoles de la région sont disposées à nous accueillir. Plusieurs enseignantes sont intéressées à s'impliquer dans le projet. De son côté, l'Université du Québec en Abitibi-Témiscaminque nous laisse une grande liberté d'action en regard de la pédagogie universitaire. Le projet a rapidement démontré des retombées intéressantes, ce qui nous a permis de bénéficier d'un soutien financier pour élaborer certains documents qui ont facilité le fonctionnement du cours.

\subsection{Pilotage du changement de pratique}

La méthodologie utilisée pour piloter le changement de pratique et pour en évaluer la progression se rapproche des recommandations de Collerette (2004), soit :

- La problématique est partagée par tous les participants : étudiants, professeure, enseignants, directeurs d'établissement ;

- L'analyse de la situation présentée aux participants permet de dégager les avantages potentiels du changement ;

- Les difficultés anticipées sont clairement énoncées et les moyens d'y remédier sont annoncés.

Lorsque le projet commence, nous mettons en place différents moyens pour favoriser les chances de réussite des étudiants dans le dispositif de formation. Pour cela, il convient de préciser quelques principes de fonctionnement :

- L'objectif général des interventions avec les élèves dans les écoles primaires est recadré chaque semaine par la professeure. Cela permet d'introduire la période de questions et de mise en commun des différentes problématiques rencontrées. Ce partage permet aux étudiants de mettre à profit leurs connaissances et leurs compétences. Ensemble, ils proposent différentes options pour intervenir auprès des élèves. La professeure anime la discussion et favorise l'émergence des solutions. Par contre, elle entre en scène lorsque la situation l'exige. Cette période dure environ 30 minutes et sert de point d'ancrage pour mettre en valeur les éléments théoriques qui suivront ;

- Les problèmes techniques, s'il y a lieu, sont rapportés lors de cette période et sont traités sur-le-champ. Les étudiants doivent mobiliser toutes leurs ressources pour y remédier. La professeure intervient au besoin ;

- La professeure est disponible lors des interventions avec les élèves. Les étudiants peuvent recourir à son expertise en tout temps ;

Revue internationale de pédagogie de l'enseignement supérieur, 25(2) | 2009 
- Des rencontres individuelles sont proposées aux étudiants qui vivent des situations particulières.

À la fin du projet, un moment est réservé à l'évaluation du projet avec les étudiants. Cette discussion est structurée et conduite de la façon suivante :

- Un retour sur les avantages du projet selon le point de vue des étudiants est effectué ;

- Les difficultés éprouvées sont relevées et les moyens d'y remédier sont proposés ;

- Une période de remue-méninges (brainstorming) est mise en place pour faire émerger des améliorations qui pourraient enrichir le projet dans son ensemble.

Une évaluation du projet avec les partenaires du milieu scolaire est aussi prévue. Elle est animée sensiblement de la même façon que celle qui est réalisée avec les étudiantes. Elle vise essentiellement à bonifier le projet.

\subsection{Difficultés rencontrées}

L'accès aux ouvrages de référence sur la didactique des mathématiques a été notre principale difficulté. En effet, les étudiants doivent rapidement consulter des documents traitant de l'enseignement et de l'apprentissage des différents concepts mathématiques pour planifier leur intervention. Nous avons donc proposé plusieurs ouvrages, mais aucun ne traite spécifiquement des difficultés d'apprentissage liées à ces concepts et encore moins des actions proposées pour y remédier. Nos étudiants n'arrivent pas à trouver les informations dont ils ont besoin d'une séance à l'autre et cette situation génère beaucoup d'insécurité. Nous avons donc rédigé des documents de référence. Ces documents sont évalués par les étudiants et améliorés d'année en année.

Une autre difficulté concerne le rôle de la professeure. Delorme, Jean \& Des Marchais (1987) rapportent que les professeurs qui s'initient à l'apprentissage par problèmes se préoccupent de leur nouveau rôle et se mettent à douter des apprentissages que leurs étudiants vont effectivement réaliser puisqu'ils sont moins présents et doivent même prendre une posture en retrait pour laisser place aux apprenants et à leurs apprentissages. Ainsi, si la professeure doit être disponible, elle n'est pas nécessairement en présence de ses étudiants. Comme le relèvent Delorme, Jean \& Des Marchais, nous avons effectivement été affectée par ce nouveau rôle. Par ailleurs, comme nos étudiants interviennent directement avec des enfants, le code d'éthique nous invite à rester présente tout au long des interventions. Cela nous permet de constater rapidement les progrès de nos étudiants et favorise notre adaptation au changement de rôle.

Parmi les difficultés relevées du côté des étudiants, signalons qu'ils doivent apprendre à gérer les nombreux déséquilibres générés par les problèmes mathématiques que l'élève dont ils ont la charge rencontre inévitablement. Ils doivent mobiliser plusieurs compétences et investir beaucoup de temps dans la préparation de leur intervention. Les discussions en groupe sont exigeantes et les étudiants doivent faire preuve d'humilité et d'honnêteté pour prendre en considération le point de vue des autres et se distancier du leur (Guilbert \& Ouellet, 1997).

Finalement, malgré les précautions prises et les nombreux retours sur les objectifs retenus pour ce cours, nous avons peu de contrôle sur les objectifs cachés que se fixent certains étudiants. En effet, lorsque l'étudiant prend conscience qu'il aide véritablement l'enfant en adoptant une approche socioconstructiviste et qu'il en 
mesure les effets positifs, il veut ensuite l'aider dans la résolution de toutes les difficultés que l'élève rencontre plutôt que de se concentrer uniquement sur celles qui ont été identifiées dans le cadre du projet. Il se fixe alors des objectifs cachés et cela augmente son anxiété face à la planification de ses interventions et face au cours d'une façon générale.

\subsection{Retombées}

Ce projet donne l'occasion aux étudiants de développer les compétences qu'exigent les interventions réelles avec des élèves en grandes difficultés, notamment la capacité à analyser les erreurs des élèves, à questionner les enfants en panne de réussite, à reconnaître les efforts de l'élève malgré ses échecs et à créer un climat d'apprentissage qui favorise la construction des connaissances. Finalement, avec ce projet, ils prennent conscience de leurs forces et cela augmente leur confiance en eux comme professionnels de l'enseignement. Ils sont plus actifs que lors des cours magistraux, car ils sont responsables des activités prévues au plan d'intervention qu'ils ont élaboré. Ils s'engagent sans compromis dans leur relation pédagogique avec l'enfant qui leur est confié. Le sentiment qu'ils aident réellement un enfant joue un rôle sur leur motivation. Soulignons que l'impact de ce type de pédagogie sur le développement des compétences est aussi reconnu par d'autres auteurs (Collerette, 2004; Guilbert \& Ouellet, 1997 ; Larue \& Cossette, 2006 ; Langevin \& Bruneau, 2000).

31 Les retombées pour la professeure concernent également l'évaluation des apprentissages des étudiants. En effet, les interventions directes lui permettent d'observer les étudiants en action et d'évaluer la progression de chacun par rapport aux objectifs du cours.

\subsection{Les limites}

Nous observons, par le biais des évaluations du cours, que les étudiants qui font partie d'un groupe d'une vingtaine de participants ou moins sont très satisfaits. Par contre, dans le cas où le groupe s'approche de 40 , le taux de satisfaction diminue. Cela va dans le sens des limites de ce type de pédagogie qui privilégie plutôt des groupes comprenant entre huit et 12 participants (Mauffet, 2003).

Le problème des documents de référence constitue à la fois une difficulté et une limite. En effet, on ne peut prévoir tout ce qui découle d'un changement de pratique. Il faut donc être disposé à se mobiliser pour faire face aux nouveaux besoins.

Nous relevons aussi des différences dans le rythme auquel les étudiants développent les compétences compte tenu de la pédagogie mise en œuvre. Cette observation est une limite rapportée dans la littérature, mais elle s'applique aussi à d'autres approches pédagogiques. Larue \& Cossette (2006) précisent que le fait d'avoir des objectifs très précis ainsi qu'une charge de travail élevée sont des éléments qui peuvent nuire au développement de stratégies d'élaboration des connaissances. Nos observations vont dans le même sens. Les objectifs cachés dont nous avons déjà parlé sont aussi à considérer. En effet, certains étudiants vivent beaucoup de pression en fonction de la réussite de l'enfant qu'ils accompagnent. Ils sont alors en surcharge cognitive, ce qui les amène à s'éloigner des intentions réelles du cours. 


\section{Conclusion}

La problématique à l'origine de ce projet peut se lire comme suit : les étudiants, futurs enseignants au préscolaire et au primaire, ont de la difficulté à élaborer et à conduire des situations d'enseignement-apprentissage dans une perspective socioconstructiviste. Leur vécu expérientiel en regard du constructivisme est quasi inexistant vu leur parcours scolaire, alors que toutes leurs interventions comme professionnels de l'enseignement devront s'orchestrer autour de cette perspective. Cet écart entre ce qu'ils ont vécu et ce qu'ils doivent faire engendre des difficultés à développer des compétences professionnelles.

Même si nous n'avions pas toutes les conditions favorables pour passer à un apprentissage par problèmes, comme la taille des groupes et le manque de documents spécialisés, nous avons proposé à nos étudiants des situations d'apprentissage authentiques qui les ont fait travailler directement auprès d'élèves du primaire. Depuis plus de dix ans maintenant, nous pilotons et évaluons ce projet avec nos étudiants et nos partenaires. Les résultats des étudiants aux examens et les travaux qui découlent des interventions nous permettent d'évaluer les compétences réelles des étudiants face aux objectifs du cours. Un certain transfert des connaissances est aussi observé. En effet, les maîtres de stage nous ont informée que nos étudiants font souvent référence à ce qu'ils ont réalisé avec leur élève pour contextualiser d'autres connaissances et qu'ils utilisent le document de référence élaboré spécifiquement pour les besoins de ce projet dans le cadre d'autres cours. Ce projet, élaboré avec nos partenaires du milieu scolaire, leur permet aussi de comprendre comment l'élève en difficulté en arrive à réorganiser ses concepts pour réussir. Le verbatim des enfants devient un repère non seulement pour intervenir sur les difficultés d'un élève mais aussi pour développer une approche préventive face aux difficultés d'apprentissage. Ces interventions sont au cœur de notre projet et permettent d'introduire le contenu théorique lié au cours.

Peut-être avons-nous cru, à un certain moment, que nous avions le choix entre une pédagogie traditionnelle et une pédagogie basée sur l'apprentissage par problèmes. Or, quand nous observons les résultats que nous obtenons aujourd'hui, il serait difficile de revenir à nos anciennes pratiques. Par contre, nous sommes consciente des caractéristiques liées à ce type de pédagogie et des conditions qui lui sont inhérentes. Il faut reconnaître que, dans certains cas, cette pédagogie est irréaliste et qu'il serait téméraire de consentir à l'instaurer.

\section{BIBLIOGRAPHIE}

Collerette, P. (2004). Programme de formation sur le pilotage du changement en milieu scolaire. Recueil inédit, Université du Québec en Outaouais.

Delorme, P., Jean, P. \& Des Marchais, J.E. (1987). Des professeurs expérimentent l'apprentissage par problèmes. L'union médicale du Canada, 116, 278-284. 
Guilbert, L. \&, Ouellet, L. (1997). Étude de cas : Apprentissage par problèmes. Sainte-Foy, Québec : Presses de l'Université du Québec.

Jonnaert, P. \&, Vander Borght, C. (1999). Créer des conditions d'apprentissage : un cadre de référence socioconstructiviste pour une formation didactique des enseignants. Bruxelles : De Boeck Université.

Larue, C. \&, Cossette, R. (2006). Apprendre en apprentissage par problèmes : un lent processus de maturation. Consortium d'animation sur la persévérance et la réussite en enseignement supérieur.Récupéré le 15 avril 2009

http://www.uquebec.ca/capres/fichiers/art_UDM_oct.06.shtml

Langevin, L. \&, Bruneau, M. (2000). Enseignement supérieur : Vers un nouveau scénario. Paris : ESF.

LeDoux, M. (2003). De la théorie à la pratique; le travail en projet à votre portée. Montréal : Les éditions CEC.

Mauffet, Y. (2003). Apprentissage par projet. Recueil inédit, Université du Québec en AbitibiTémiscamingue.

Piaget, J. (1975). L'équilibration des structures cognitives; problème central du développement. Paris : Presses Universitaires de France.

Roy, A. (2007). Liens entre les représentations idéologiques des étudiants-maîtres et leurs habiletés réflexives en éducation. Communication présentée au $1^{\mathrm{er}}$ séminaire international de didactique des mathématiques à l'UQAT, Rouyn-Noranda, Québec, mai 2009.

\section{RÉSUMÉS}

En tant que professeure à la formation des maîtres, nous constatons qu'il est difficile pour nos étudiants d'adhérer à l'enseignement des mathématiques dans une perspective socioconstructiviste. Cela peut s'expliquer, en partie, par le fait que, dans leur parcours scolaire, ces étudiants ont vécu peu d'expériences de ce genre. Leur propre apprentissage des mathématiques a surtout été centré sur des explications et des démonstrations. De ce fait, ils tendent à reproduire ces comportements malgré les théories socioconstructivistes qui leur ont été préalablement enseignées. L'apprentissage par problèmes nous a servi de cadre de référence pour développer, en partenariat avec deux écoles primaires, un projet où les étudiants interviennent directement auprès d'enfants en grande difficulté d'apprentissage en mathématiques. Ces rencontres modifient le répertoire expérientiel des étudiants et deviennent des points d'ancrage pour planifier et piloter des situations d'enseignement-apprentissage qui favorisent la construction des concepts mathématiques chez les enfants.

As a professor in teacher training, we noticed that our university students had difficulties in teaching mathematics from a socioconstructivist perspective. This can be explained, in part, by the fact that during their schooling, these students have had few experiences of this type of teaching. Their own learning in mathematics consists mostly of explanations and demonstrations. They tend to reproduce these patterns, in spite of the socioconstructivist theories they are introduced to. A teaching approach based on problem solving was used as a framework for developing a project, in partnership with two primary schools, where student teachers tutored children experiencing significant learning difficulties in mathematics. These tutorials modified the students' experiential repertoire and became a framework for planning and supervising teaching and learning activities which encourage children to construct mathematical concepts by themselves. 
INDEX

Mots-clés : apprentissage par problèmes, changement de pratique, didactique des mathématiques, socioconstructivisme

Keywords : change in practice, didactics of mathematics, problem-based learning, socioconstructivism

\section{AUTEUR}

\section{COLETTE PICARD}

Professeure en didactique des mathématiques

Département des sciences de l'éducation

Université du Québec en Abitibi-Témiscamingue

$6751^{\text {re }}$ Avenue, Vald'Or J9P 1Y3, Québec

Courriel : colette.picard@uqat.ca 